\title{
Python 之提速与深化教学
}

\author{
李飞 \\ 浙大城市学院 \\ DOI:10.32629/mef.v3i4.791
}

\begin{abstract}
[摘 要] Python具有简单易懂和功能强大的特点, 目前, Python在企业和学校都普及起来。做好Python 的教学工作不仅有关高等院校的自身建设问题，也是国家信息化战略的重要一环。而目前高校Python 的教学仍沿用过去C语言、VB语言等老的编程语言的教学方式：对基础部分过多地讲授。这种教学方 法不符合Python的自身特点, 也不利于学校 “多出人次, 快出人才” 指导方针。新的教学方式从Python 的自身特点出发, 论述Python教学中的提速与深化并举的可能性与必要性, 从: 1) Python教学内容的 广度拓宽, 2 ) Python进阶部分的深度加强, 3 ) 各章节（包括基础与进阶部分）的理论与案例设置与 讲解，4）课堂讲授、作业、考核方式的设计四个方面，阐述在Python教学中的这一新的教学方法。
\end{abstract}

[关键字] Python; 教学; 案例; 广度; 深度

\section{Speeding up Python and Deepening Teaching}

Fei Li

Zhejiang University City College

[Abstract] Python is easy to understand, and is powerful. At present, Python is popular in both enterprises and schools. Doing a good job of teaching Python is not only related to the self-construction of colleges and universities, but also an important part of the national informatization strategy. At present, the teaching of Python in colleges and universities still follows the teaching methods of the old programming languages such as C language and VB language, which is taught to the basic part too much. This kind of teaching method does not conform to Python's own characteristics, and is not conducive to the school's guidelines of "get talent more, get talent quicker". The new teaching method is based on Python's own characteristics, discusses the possibility and necessity of speeding up and deepening simultaneously in Python teaching, from: 1) Widening of Python teaching content, 2) Deepening of the advanced part of Python, 3) The theory and case setting and explanation of each chapter (including the basic and advanced parts), 4) Four aspects of the design of classroom teaching, homework, assessment methods, and elaborates this new teaching method in Python teaching.

[Key words] Python; teaching; cases; breadth; depth

\section{Python 教学的现状分析}

目前, Python以其方便灵活和功能 强大的特点, 在全世界广泛流行。按照 IEEE Spectrum的统计: Python、Java和 C++是2017年最流行的编程语言, 各大 公司包括 Google、IBM 等都在使用 Python, 因此Python的市场需求非常庞 大。鉴于此, 高校将Python作为主要的 教学语言, 取代原有的VB、PhotoShop 等课程教学; 社会上有各种各样的培训 机构将Python列为主要的职业培训内 容; 中等义务教育也在2017年将Python
的教学下放到高中信息课程中。总之, Python教学在高校、中学和社会上正越来 越普及和广泛。但是, 目前在高校, 很 多人对Python教学的认识, 还停留在它是 对过去VB、PhotoShop等课程的替代教 学, 基本教学方式也是沿袭以前的 $\mathrm{C} 、$ VB语言的教学方法: 从常量-->变量--> 结构-->组合数据类型-->函数-->文件, 有 时可能会相对深入一些, 即结合算法讲 解这些概念, 但也以基本概念为主。这 样的教学模式有这样几个特点: 1) 非常 注重基础的练习, 对基本概念掌握牢固;
2) 老师对学生的授课基本是单向输出 的, 学生掌握基本概念, 也就基本完成 教学目标; 3) 该教学方法几乎是静止的, 没有学生问题或者实际应用需求反哺教 学的进步; 4) 整体教学工作量偏少。

2 教学方法改进的必要性与可 行性分析

这种教学方法, 在Python这种新的 信息技术形态下, 存在这样几个缺陷:

2.1 学生完全不能体会Python的强 大, 对于现阶段国外、国内普及Python 教学的重要性不能理解, 不少同学认为 
Python教学只不过是换了门语言而已。

2.2学生学习容量不够, 没有看到 Python基础知识只是Python应用很小的 方面, 在其后面还有丰富的宝藏, 现在 高校也在普及人工智能的教育, 而当前 机器学习、大数据处理的语言又基本使 用Python, 很多同学不得不在选修这些 课的时候, 重修Python或者放弃这门课。

2.3 离应用还有不少差距, 目前计算 机系的学生基本还是使用Java，毕业以 后, 面临数据分析等工作需求的时候, 还要进行职业再教育。

2.4 学生也不能体会信息技术的最 新进展, 无法感受信息技术的日新月异。 Python本身技术在发展，还有很多的第 三方库也在进化，互联网上有诸多的用 Python技术的应用，但学生们反而被 Python隔离在实际应用以外。

上述的分析表明: Python的教学必 须遵循其自身的规律, 要像我们学习自 然语言一样, 既要讲解基础知识, 也要 在应用中进行学习，而不是把应用和基 础部分离开来。我们认为: Python教学 中将基础教学与应用开发这两个方面结 合起来，在广度和深度方向拓展也是可 行的。首先: Python的基础知识部分已 经极大地简化, Python诞生之初, 就是 为非计算机专业人士使用的语言, 其尽 量接近自然语言的特点, 使得程序阅读 不再费力。在教学中, 基础部分教学也 可以弱化，关键是在应用中揣摩和辅助 一定的讲解, 最终熟能生巧。其次, Python的深度也可以加强, 现阶段高校 对C、Java的教学基本是还是按照非对象 编程与面向对象的思想两个方向展开 的, 而Python却是完整的面向对象程序 设计, 特别是在应用中, 有的是调用第 三方库中的类或对象等, 所以Python的 教学实际应该以面向对象方法为主, 这 样在以后面向Python实际应用的时候, 学生已经具备了面向对象的编程基础。

\section{Python 教学中的具体方法}

3.1扩充原有Python的教学内容, 内 容基础上, 增加各个领域的应用, 包括: 面向对象编程、GUI编程、游戏、动画 游戏、网络、数据分析、Web编程和数
据库编程。原有的课程内容在总体在7-8 节, 现在将教学内容扩充到 15 节: 基础 部分6章, 面向对象编程 2 章和上述 7 个方 面的应用7章。

3.2增加原有Python教学深度, 将原 来Python的基础部分由原来的9章压缩 到6章, 有些基础内容比如组合数据类 型, 原来都是分为2-3章讲授, 现在合并 为一章, 同时, 增加面向对象的编程内 容, 讲解Python的类定义、封装、继承 和多态的概念, 将面向对象的编程内容 放在两个章节中, 并且以两个案例贯穿 全部知识的讲解。

3.3 以案例为主线, 贯穿Python在每个 应用领域的编程, Python语言简单, 也有 大量的第三方库, 目前绝大多数教材中, 从介绍第三方库开始, 到类的使用, 再介 绍一个应用, 这种方法, 虽然对某类库可 以全面掌握, 但是学生的学习没有目的 性, 学习过程也索然无味和容易疲劳。我 们为每一章配一个趣味案例, 比如, 在面 向对象编程中, 使用 “黑Jack 21点游戏” 为案例, 在普通游戏和动画游戏中, 分别 以 “疯狂Pizza” 和 “雷霆战机” 两个游戏 为案例, 其他各章还设计有 “决策树分 析”、“影评网页爬取与分析” “邮件发送” 案例等等, 通过案例的程序解读, 使学生 掌握Python编程语法。

3.4确定课堂讲授方式、课堂作业与 课程考核。上述案例的课堂讲解也是循序 渐进的: 首先给学生演示这个案例的总体 效果, 使学生对应用领域、对程序效果有 总体的印象, 接着搭建一个整体框架, 然 后像搭积木一样, 一个模块一个模块填 充。即使在前六章的基础部分, 也尽可能 以案例讲解为主, 比如用一个学生课程成 绩统计表格进行成绩分档的例子, 讲解文 件操作、组合数据类型操作等。每一章都 布置课堂作业, 课堂作业主要考察学生对 案例的理解, 作业基本内容是修改或者增 加所讲授案例的功能, 要求学生当场提交 修改程序, 在我校网络教学平台上统计得 分, 并最后计入期末总评成绩。学期总体 考核分为 3 个部分: a)期中考试, 主要在基 础部分结束以后, 用课堂时间当场编写程 序, 内容为批量数据处理, 涵盖文件操作
和组合数据操作; b)平时课堂作业; c)期 末大作业, 以一个综合的应用程序, 改写 功能或者解释功能。

\section{4 教学效果}

我校从2016年开始以通识公选课的 形式试点Python教学, 到2019-2020第一 学年, 已经进行了四轮Python教学, 现 在分为两个班: Python基础程序设计, 2.0 学时, 和Python程序与应用开发, 3.0 学时, 前者以目前的教材, 讲解基础部 分为主, 后者采用本文的方法。

从学生选课人数来看, 采用本文 方法的 “Python程序与应用开发” 的 人数逐年增加, 四个学期的教学中, 人数大致在 $30 、 30 、 60 、 80$ 人, 反映 出学生对Python的学习兴趣, 也意思 到Python的重要性。其次, 从学生的 组成来看, 选课学生绝大部分来自计 算学院和工程学院, 他们的学习该课 程的目的比较明确: 希望通过一个学 期的学习, 能极大地提高自己的编程 水平。在期末, 作为考察手段, 我们 要求学生解析一个应用的Python代码或 者自己编写一段程序。有很多学生选择 了游戏和数据爬虫代码, 从答辩中, 我 们看出他们已经能完全理解面向对象的 游戏和Web编程程序。有个别优秀的学 生, 独立编写了 700/800多行的校园学生 抢课程序。他们也一致反映: 本课程的 信息量大, 新的教学方法学起来不累, 而且一个学期下来，很有收获。

\section{5 总结}

由于教学资源的丰富及各种教学手 段的普及, 作为程序设计语言教学之一 的Python教学, 也面临新的挑战, 在需 求快速变化的今天, 强调基础、沉稳缓 慢的教学节奏，有时候不太适应形势的 变化。按照抓住主线、带领学生高屋建 瓴地学习程序语言地思路, 我们进行了 Python程序与应用开发的教学探索, 从 实际效果来看, 这种方法能起到事半功 倍的作用。

\section{基金项目:}

教育部产学合作、协同育人项目 《面向互联网大数据的本科理论与实 践新教学探讨》(编号：201902230004)。 


\title{
高校教学诊改实践探究
}

\author{
吴旭光 \\ 浙江国际海运职业技术学院 \\ DOI:10.32629/mef.v3i4.801
}

\begin{abstract}
[摘 要] 本文以高校教育教学为研究对象, 在深化高校教育改革、提升人才培育质量的目标引导下, 提出了高校教学诊改规划。在结合对目前高校教育教学整体状况的综合研究与分析, 在对高校教学诊 改意义进行探究的基础上, 揭示目前高校教学存在的现实问题, 并以此为基础提出切实可行的教学诊 改实践策略。希望能够借助以下研究和探讨, 使高校在教学诊改的作用下, 完成对自我教育教学工作 一次完整、全面、充分的检阅，为高校教育教学质量的提升奠定坚实的基础。
\end{abstract}

[关键词] 高校; 教育教学; 教学诊改; 现实问题; 实践策略

\section{A Probe into the Practice of Teaching Diagnosis Reform in Colleges and Universities}

\author{
Xuguang $\mathrm{Wu}$
}

Zhejiang International Maritime College

[Abstract] Taking college education as the research object, and under the guidance of the goals of deepening the reform of college education and improving the quality of talent cultivation, this article proposes a teaching reform plan of colleges and universities. Combined with the comprehensive research and analysis of the college education and teaching at present, on the basis of exploring the significance of college teaching diagnosis reform, it reveals the current practical problems in college teaching, and put forward practical strategy of teaching diagnosis reform based on it. It is hoped that with the help of the following research and discussion, colleges and universities can perform a complete, comprehensive and sufficient review of self-education teaching under the role of teaching diagnosis reform, and lay a solid foundation for the improvement of college education and teaching quality.

[Key words] colleges and universities; education and teaching; teaching diagnosis reform; reality; practical strategy

一直以来党中央政府都十分关注我 国教育事业的发展, 将教育强国作为实 现中华民族伟大复兴的基础工程, 立足 于 “深化教育改革, 加快教育现代化,
办人民满意的教育”。虽然我国高校教育 一直以来都在进行着一系列的教育改 革, 实现了教育教学质量的明显提升。 但受各方面现实因素的影响, 我国高校
教育在发展中仍存在不少问题, 《国家职 业教育改革实施方案》就在内容中明确 指出 “我国职业教育还存在着体系建设 不完善、职业技能实训基地建设有待加

\section{[参考文献]}

[1]嵩天, 黄天羽.Python 语言程序 设计教学案例新思维 [J]. 计算机教 育,2017(12): 11-19.

[2]郑世秀,刘晓洁.从计算思维视角 看通识教育中程序设计类课程的选择 [J].教育教学论坛,2019(3):265-267.

[3]李博,董付国,冯烟利,等.面向软 件工程专业的Python 课程建设 [J]. 计 算机教育,2019(1):92-96.
[4]教育部高等学校大学计算机课 程教学指导委员会. 大学计算机基础课 程教学基本要求 [M]. 北京: 高等教育出版 社,2017.

[5]魏冬梅, 王秀华, 王影, 等. 基于 Python 的程序设计通识课程建设与教 学实践[J].计算机教育,2019(2):69-73.

[6]许朝侠. 非计算机专业Python语 言程序设计教学体系建设初探 [J]. 软件 导刊・教育技术,2019(2):48-50.
[7]刘培刚, 杨劯辉, 李昕. 应用导向 的新工科大学计算机课程建设探讨 [J]. 软件导刊,2019(2):186-189.

[8]杨荣领.《Python语言程序设计 基础》课程教学改革实践 [J]. 高教导 刊,2019(3):135-137.

\section{作者简介：}

李飞(1972--), 男, 汉族, 安徽 黄山人, 讲师, 博士, 研究方向: 机器 学习。 